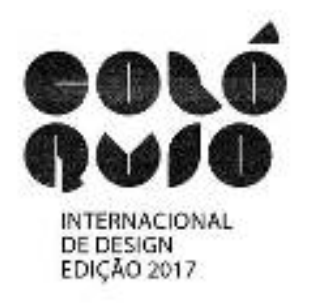

\title{
Descrição e Análise das Técnicas de Prototipagem no curso de Design da Unesp de Bauru
}

\author{
João Victor Gomes dos Santos; \\ Michel Silvestre de Souza; \\ Osmar Vicente Rodrigues
}

resumo:

A prototipagem virtual tem revolucionado o Processo de Desenvolvimento de Produtos, possibilitando a produção de peças físicas complexas e detalhadas através de modelos virtuais, obtendo grandes vantagens principalmente na redução do tempo e inovação. No meio acadêmico estes softwares de modelagem virtual aparecem de forma lenta devido à falta de infraestrutura, resultando em alunos com conhecimento defasado. As novas técnicas de prototipagem, assim como as convencionais, são importantes para o desenvolvimento dos profissionais das áreas relacionadas à inovação e desenvolvimento de novos produtos. Portanto, o intuito do presente trabalho é desenvolver um material que sirva de referência para profissionais e estudantes da área. Para tanto, foi realizado um levantamento dos métodos e materiais convencionais e contemporâneos de modelagem e prototipagem do curso de Design da FAAC - UNESP, campus de Bauru. Através de consultas a materiais bibliográficos disponíveis, professores e técnicos atuantes no setor, foi realizada uma coleta documental com fotos de equipamentos, processos e dados sobre os métodos e materiais utilizados para modelagem e prototipagem, obtendo um panorama da atual infraestrutura dos laboratórios, das técnicas e tecnologias utilizadas no curso e suas respectivas particularidades. Por se tratar de uma pesquisa focada no design de produtos, foram abordados apenas os laboratórios ligados ao desenvolvimento de modelos e protótipos sendo eles: Laboratório Didático de Materiais e Protótipos (LDMP), do Centro Avançado de Desenvolvimento de Produto (CADEP), o Laboratório de Processamento de Madeira e Bambu (LPMB) e do Laboratório de Ergonomia e Interfaces (LEI). Os resultados desta coleta documental foram organizados em tópicos cuja disposição segue uma ordem cronológica a partir da fundação do curso de Design da referida universidade, a criação dos laboratórios e finalizando com a descrição das metodologias e técnicas de prototipagem utilizadas.

\section{palavras-chave:}

Prototipagem rápida; impressão 3D; Design; Unesp; Bauru 


\section{Introdução}

Soluções dinâmicas como os softwares CAD (Computer Aided Design) e técnicas como prototipagem virtual vêm revolucionando o Processo de Desenvolvimento de Produtos (PDP) das indústrias. Além de possibilitar a produção de modelos e protótipos diretamente a partir do modelo virtual 3D, é possível obter peças físicas acabadas de modo automático com complexidade e detalhes que não seriam possíveis pelos sistemas convencionais de desenho técnico ou tornariam sua execução excessivamente demorada. Esses avanços constituem uma vantagem considerável para a indústria, uma vez que, com o emprego dessas tecnologias, há uma maior agilidade e eficiência no PDP que resulta também na redução do tempo de lançamento de novos produtos (ROZENFELD, 2006).

De acordo com Rodrigues (2011), no meio acadêmico essa realidade não é diferente. As vantagens das novas tecnologias de prototipagem também podem ser observadas nas atividades de ensino e pesquisas relacionadas ao PDP. A produção de alternativas, controle da evolução das ideias, elaboração de projetos executivos e diversas outras etapas do PDP são visivelmente potencializadas com os programas de modelagem virtual paramétrica.

Contudo, de acordo com o autor, essas recentes ferramentas digitais de desenho por computador trazem também um efeito negativo. Além do pouco contato com disciplinas relacionadas ao desenho antes da universidade, o acesso dos discentes a softwares cada vez mais interativos e capazes de realizar operações de maneira mais rápida, reduz a necessidade de se preocupar com procedimentos que, anteriormente, dependiam integralmente da capacidade de visualização e interpretação do profissional.

Além disso, Forti (2005) afirma que a utilização desses softwares no meio acadêmico aparece de forma lenta. Ainda segundo o autor, a falta de infraestrutura e de atualização dos professores faz com essas novas metodologias e tecnologias não consigam substituir o ensino das antigas práticas de desenvolvimento de projeto, resultando em alunos com conhecimento defasado e, portanto, mal preparados para o mercado.

Rodrigues (2011) concluiu em seu experimento que a materialização de objetos e produtos, através de qualquer processo de modelagem física, pode funcionar como ferramenta de desenvolvimento da inteligência, particularmente a inteligência criativa, melhorando o nível de entendimento consciente do processo criativo e a habilidade de comunicá-lo. Ou seja, de desenvolver um "Design Thinking" próprio a respeito.

Diante do contexto apresentado conclui-se que o domínio, tanto das técnicas convencionais quanto das modernas, são fundamentais para o desenvolvimento do designer. Portanto, no intuito de desenvolver um material que sirva de consulta e referência para futuras pesquisas deste âmbito, procurou-se realizar um levantamento dos métodos e materiais convencionais e modernos para modelagem e prototipagem do curso de Design da Faculdade de Arquitetura, Artes e Comunicação da UNESP, campus de Bauru. Para tanto, foi consultado o material bibliográfico disponível desde a inauguração do curso bem como professores e técnicos atuantes no setor.

\section{Prototipagem}

O Processo de Desenvolvimento de Produtos é composto por diversas etapas que devem ser constantemente avaliadas para garantir que o produto desenvolvido atenda os objetivos inicialmente propostos pelo projeto (BAXTER, 2011). Para tanto, Lopes e Naveiro (1998) consideram essencial a construção de modelos com os quais seja possível executar testes que validem as capacidades que o produto em desenvolvimento deve responder segundo as exigências para as quais foi projetado.

Dessa maneira, de acordo com Lesko (2012), se o designer é responsável pela aparência e forma de um produto, por exemplo, é de suma importância que tenha conhecimento a respeito dos materiais, metodologias e processos de fabricação empregados para a construção desse produto, já que suas características estão diretamente associadas a estes elementos. Logo, quanto maior o repertório do designer maior a gama de soluções e potencial criativo com os quais poderá trabalhar.

Fatores como tempo, infraestrutura e recursos financeiros, devem ser analisados antes de optar entre a confecção de um simples modelo do produto final, ou seja, uma amostra de baixa fidelidade cujo material, funcionalidade e escala são diferentes do produto final; ou um protótipo, definido como 
um exemplar de alta fidelidade do produto final (mesmas funcionalidades, materiais, escala e processo de produção). Por se tratar de um produto mais complexo, o protótipo demanda mais recursos, portanto, antes de determinar sua confecção, é necessário considerar também a sua relevância. Em vista disso, os protótipos são geralmente as versões finais apresentadas após todo o Desenvolvimento do Projeto, já que nesta etapa do PDP presume-se que existência de falhas seja consideravelmente menor. Este é um fator importante, visto que o propósito do protótipo é validar e homologar um produto através de testes de funcionamento, ou seja, avaliações de montabilidade, desempenho e concordância com as especificações iniciais do projeto (ROZENFELD, 2006).

Além dos tipos já citados, os modelos e protótipos podem ainda ser classificados em físicos, ou seja, elementos tangíveis que possibilitam avaliar aspectos como ergonomia e estabilidade, ou virtuais (intangíveis) que geralmente são aplicados em situações nas quais a prototipagem física é inviável ou dispensável (GRIMM, 2005). Após os testes piloto, se aprovado, o produto é encaminhado para a linha de produção. Caso contrário, o produto é reformulado com o intuito de corrigir as possíveis falhas e novas avaliações são conduzidas. Este ciclo pode ser repetido até que o produto seja aprovado para produção.

\subsection{Técnicas convencionais e sua importância}

Em um experimento realizado com profissionais e não-profissionais do setor de modelagem, Rodrigues (2011) constatou que, devido a limitação em se fazer uma boa leitura de um determinado desenho, tanto os profissionais quanto os não-profissionais apresentaram dificuldades na construção de modelos físicos e protótipos de um determinado produto. A dificuldade na transposição correta das informações bidimensionais para informações tridimensionais a serem aplicadas nos modelos estavam relacionadas com a má interpretação dos desenhos disponibilizados para apoio na construção do modelo.

Segundo Rodrigues (2011), a visível dificuldade apresentada pelos participantes do experimento mostra que o atual domínio digital nas atividades de desenvolvimento de um produto tem reduzido cada vez mais a capacidade de profissionais e estudantes de design de enxergar um objeto a partir de um simples desenho. Mais importante ainda, a capacidade de visualizar o resultado de qualquer operação num modelo, antes de sua execução.

Knight (2004) complementa ao afirmar que nas representações com meios analógicos o designer recorre à experiência dos sentidos ao utilizar o corpo para compreender e controlar as ferramentas que usa para representar. Já nas representações digitais, o designer não tem meios para compreender conceitos do mundo real, como a natureza do objeto ou a sua função, limitando-se apenas à capacidade de codificar conceitos geométricos. Esta afirmação é reforçada por Pereira (2015) ao afirmar que, apesar de reduzir custos e tempo nas diversas etapas do PDP, as técnicas digitais de representação de um produto dificultam a sua percepção tátil. Assim, não há a sensação de tocar e sentir a superfície e as formas produzidas no produto, dificultando a compreensão tridimensional geométrica do mesmo.

Para concluir, Forti (2005) considera que o aprendizado das novas tecnologias foi desvinculado do ato de projetar, ou seja, o aluno aprende algumas funcionalidades do software, mas não a raciocinar de maneira a utilizá-lo para desenvolver projetos e se expressar. O que pode acarretar em uma futura dificuldade do designer ao se deparar com a realidade do mercado.

É importante, dessa maneira, abordar, não somente o desenvolvimento de produtos em design com auxílio das novas tecnologias, mas também, através de uma ótica multidisciplinar, articular e integrar metodologias convencionais à atividade projetiva com os conteúdos que exploram as características específicas dos materiais.

Um exemplo são as atividades práticas em oficina que permitem apresentar ao aluno as tecnologias de prototipagem disponíveis como a prototipagem tradicional (modelagem, pintura, marcenaria e serralharia); prototipagem rápida (digitalização, impressão, fresagem e roteamento 3D) e; prototipagem virtual (projeto gráfico e interfaces). Práticas que permitem que aluno explore as propriedades e a plasticidade dos materiais na produção de objetos e aumente, dessa maneira, seu repertório e potencial criativo. 
Além dos benefícios apresentados, Nishimura et al. (2016) afirmam que essa combinação das duas técnicas, convencionais e contemporâneas, contribuem também para a otimização do PDP. Os autores sugerem que as técnicas convencionais sejam aplicadas nas primeiras fases do PDP através da elaboração de modelos de baixo custo que possibilitam testar aspectos físicos do produto, como, por exemplo, sua ergonomia. Já nas fases finais do PDP, os autores recomendam que essa peça produzida seja digitalizada através de um scanner tridimensional e, depois de realizadas as edições necessárias em programas de modelagem virtual, a peça seja fabricada com emprego de máquinas de PR, tanto aditivas quanto subtrativas. Dessa maneira, é possível conciliar os pontos positivos das duas técnicas e suprimir as deficiências, resultando em um PDP mais preciso.

\section{Método}

O método de pesquisa utilizado para este trabalho de caráter exploratório foi um levantamento de dados de natureza descritiva com o objetivo de analisar e divulgar a infraestrutura dos laboratórios do curso de Design da Unesp de Bauru. Para tanto foi realizada uma coleta documental através de consulta aos bancos de dados bem como entrevistas não-estruturadas com os professores e responsáveis pelos laboratórios com o objetivo de coletar informações e imagens das infraestruturas e materiais utilizados nestes laboratórios.

Para este tipo de obtenção de dados Lakatos e Marconi (1995) recomendam a utilização de três procedimentos: pesquisa documental, pesquisa bibliográfica e contatos diretos.

A pesquisa bibliográfica (apanhado geral sobre os principais trabalhos já realizados) fornece dados relevantes relacionados ao tema. $\mathrm{O}$ estudo da literatura pertinente pode ajudar a planificação do trabalho, evitar publicações e certos erros, e representa uma fonte indispensável de informações.

A investigação preliminar (estudos exploratórios) deve ser realizada através de dois aspectos: documentos e contatos diretos. Os principais tipos de documentos são:

a) Fontes Primárias - dados históricos, bibliográficos e estatísticos; Informações, pesquisas e material cartográfico; Arquivos oficiais e particulares; Registros em geral; Documentação pessoal (diários, memórias, autobiografias); Correspondência pública ou privada; etc.

b) Fontes Secundárias - imprensa em geral e obras literárias.

Os contatos diretos, pesquisa de campo ou de laboratório, são realizados com pessoas que podem fornecer dados ou sugerir possíveis fontes de informações úteis. As duas tarefas, pesquisa bibliográfica e de campo, podem ser executadas simultaneamente.

\section{Resultados}

A consulta à base de dados da universidade e as entrevistas não estruturadas com professores e técnicos responsáveis pelos laboratórios resultaram em informações sobre os locais, métodos e materiais utilizados para modelagem e prototipagem no curso de Design da Faculdade de Arquitetura, Artes e Comunicação da UNESP. As informações referentes aos laboratórios estão apresentadas em seus respectivos tópicos. Já os dados referentes aos materiais e métodos de modelagem e prototipagem estão reunidos, organizados e resumidos em uma tabela de fácil leitura. Juntamente com essas informações, são apresentadas fotos dos laboratórios, dos equipamentos e de alguns protótipos produzidos por alunos do curso.

\subsection{Infraestruturas dos laboratórios}

Os laboratórios apresentados neste trabalho têm sua origem na criação da UNESP (Universidade Estadual Paulista "Júlio de Mesquita Filho") em 15 de agosto de 1988, após aprovação pelo Conselho Universitário, o Governo do Estado de São Paulo incorporou a Universidade de Bauru à Universidade Estadual Paulista "Júlio de Mesquita Filho" (decreto no. 28.682) com a seguinte estrutura acadêmica: Faculdade de Arquitetura, Artes e Comunicação, Faculdade de Ciências e Faculdade de Engenharia e Tecnologia. Então, após reestruturação dos cursos de bacharelado em Desenho Industrial e 
Comunicação Visual, começou a vigorar o curso de Desenho Industrial, com as Habilitações em Projeto do Produto e Programação Visual conforme Resolução CFE no. 02 de 16 de junho de 1987 (FAAC, 2017a)

Em 2007,com a implantação do novo currículo, o curso de Desenho Industrial passa a ter a denominação de Curso de Design, oferecendo as habilitações em Design Gráfico e Design de Produto. Atualmente são oferecidas 55 vagas para Programação Visual (25 diurnas e 30 noturnas) e 30 para Projeto de Produto (noturnas).

Hoje os alunos contam com laboratórios didáticos de Fotografia, Informática, Laboratório Didático de Materiais e Protótipos, Laboratório de Pesquisa, Ensino e Extensão em Design Contemporâneo e o Laboratório de Extensão Didático Digital. Além desses, o curso de design conta ainda com três laboratórios de pesquisa e extensão: o Laboratório de Ergonomia, o Inky Design e o Centro Avançado de Desenvolvimento de Produto.

Neste trabalho serão abordados apenas os laboratórios ligados ao desenvolvimento e análise de produtos através de atividades de modelagem, prototipagem e usabilidade. Dessa forma, nas próximas subseções tem-se o detalhamento do Laboratório Didático de Materiais e Protótipos (LDMP), do Centro Avançado de Desenvolvimento de Produto (CADEP), o Laboratório de Processamento de Madeira e Bambu (LPMB) e do Laboratório de Ergonomia e Interfaces (LEI).

\subsubsection{Laboratório Didático de Materiais e Protótipos}

O Laboratório Didático de Materiais e Protótipos (LDMP) iniciou suas atividades em 1978 junto ao curso de Design da FAAC/UNESP. Atualmente as disciplinas ministradas no laboratório são: Oficina de Madeira, Modelagem e Oficina de Materiais Plásticos. O LDMP (Figura 1) oferece infraestrutura para a construção de modelos e protótipos para as disciplinas de projeto, para os TCCs e para os inúmeros concursos de Design no âmbito nacional e internacional. Apoia também atividades de pesquisa e de extensão universitária desenvolvidas na UNESP (FAAC, 2017b).

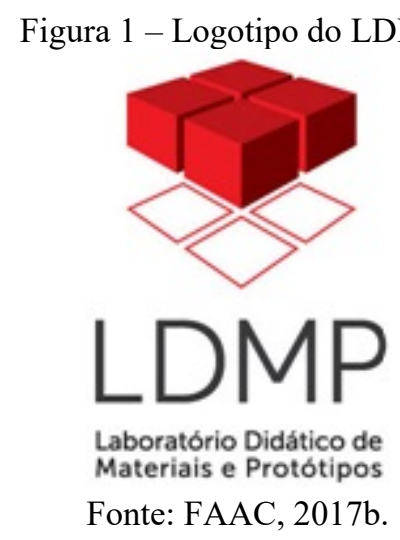

Além das ferramentas básicas de marcenaria e de EPI's (Equipamento de Proteção Individual) os alunos têm à sua disposição diversos equipamentos como serra de disco, furadeira vertical de bancada, furadeira horizontal estacionária, lixadeira de cinta estacionária, lixadeira de disco estacionária, vaccum forming, serra tico-tico estacionária, serra circular estacionária, moto esmeril elétrico, máquina policorte, serra circular esquadrejadeira, serra de fita estacionária, desempenadeira, tupia estacionária, desengrossadeira, soprador térmico e outras ferramentas convencionais de marcenaria. O laboratório conta ainda com um amplo espaço e dez bancadas de marceneiro para conforto dos alunos e professores que também dispõe de um técnico responsável pelo laboratório.

O prédio foi especialmente projetado para atender as necessidades dos alunos de graduação e pós-graduação, resultando em um ambiente seguro, ambientalmente correto, com estrutura e iluminação adequadas, e espaço de movimentação ergonomicamente dimensionado para 30 alunos por turma conforme apresentado pela Figura 2 (FAAC, 2017b). 
Figura 2 - Foto das instalações do LDMP.

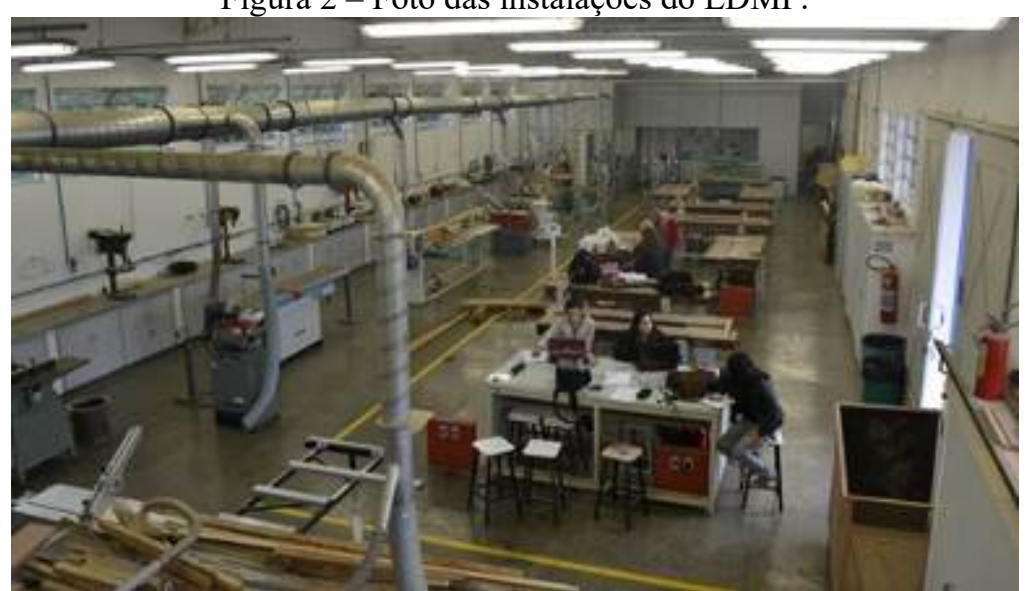

Fonte: FAAC, $2017 b$.

\subsubsection{Centro Avançado de Desenvolvimento de Produtos - CADEP}

Inaugurado em novembro de 2012, o CADEP está voltado, fundamentalmente, à pesquisa e à prestação de serviço, oferecendo também apoio à docência (graduação e pós-graduação), nas áreas de Prototipagem Rápida, Manufatura Rápida, Digitalização 3D, Inspeção Tridimensional (FAAC, 2017b).

Figura 3 - Logotipo do CADEP.

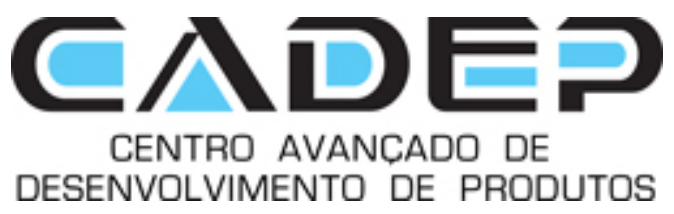

Fonte: FAAC, 2017b.

Este centro é uma iniciativa multidisciplinar, com suas tecnologias aptas a atender demandas da própria Unesp e da indústria. Além disso, o CADEP (Figura 4) se destaca pela inovação, posicionando a Unesp, desde sua fundação, como uma das 70 universidades do mundo a utilizar os tipos de tecnologias digitais de Prototipagem Rápida. Destaca-se também pelo pioneirismo, sendo um dos únicos laboratórios do gênero no mundo a combinar tecnologias convencionais (baseadas na subtração, fusão e conformação de materiais), com tecnologias digitais mais recentes (baseadas na adição de materiais), para a construção de modelos e protótipos.

Figura 4 - Fachada do Centro Avançado de Desenvolvimento de Produtos - CADEP - UNESP Bauru.

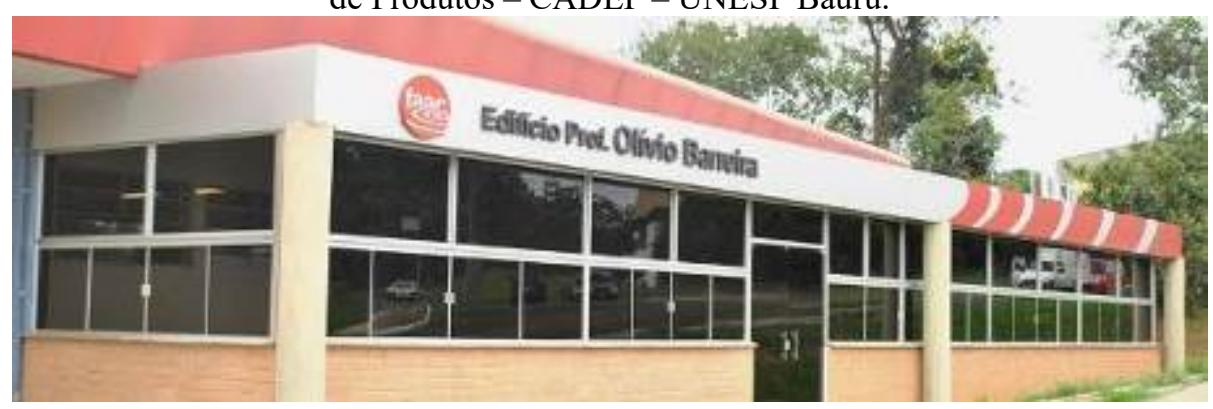

Fonte: CADEP, 2017.

O objetivo maior do CADEP para com a docência, além de, evidentemente, apoiar os alunos de graduação e pós-graduação na produção de seus modelos e protótipos, é apresentar, na prática, as vantagens, desvantagens e especificidades das tecnologias aditivas da prototipagem rápida (CADEP, 2017). 
O centro está localizado em frente ao LDMP como apresentado pela Figura 5 e oferece diversos equipamentos de informática os quais são utilizados por alunos e pesquisadores. Dentre esses equipamentos podemos listar computadores e notebooks que possuem softwares como SolidWorks, 3DS Max e Rhinoceros, dentre outros. Além de computadores, o centro de pesquisa conta ainda com uma fresadora CNC (Comando Numérico Computadorizado) Roland MDX $540 \mathrm{com} 4^{\circ}$ eixo rotacional ZCL 540, um sistema ótico de digitalização 3D móvel GOM Atos I 2M (Sala 02 - recepção), uma injetora de Poliuretano Transtécnica T-15/2 (Sala Injetora de PU), uma máquina de prototipagem rápida Z-Builder Envision (Sala ZBuilder), um router CNC 3D Transform 2000/2 (Sala Router) e uma máquina de prototipagem rápida Z-Printer 650 Z-Corporation (Sala 1).

Figura 5 - Planta baixa da estrutura do LDMP (cinza) e do CADEP (azul).

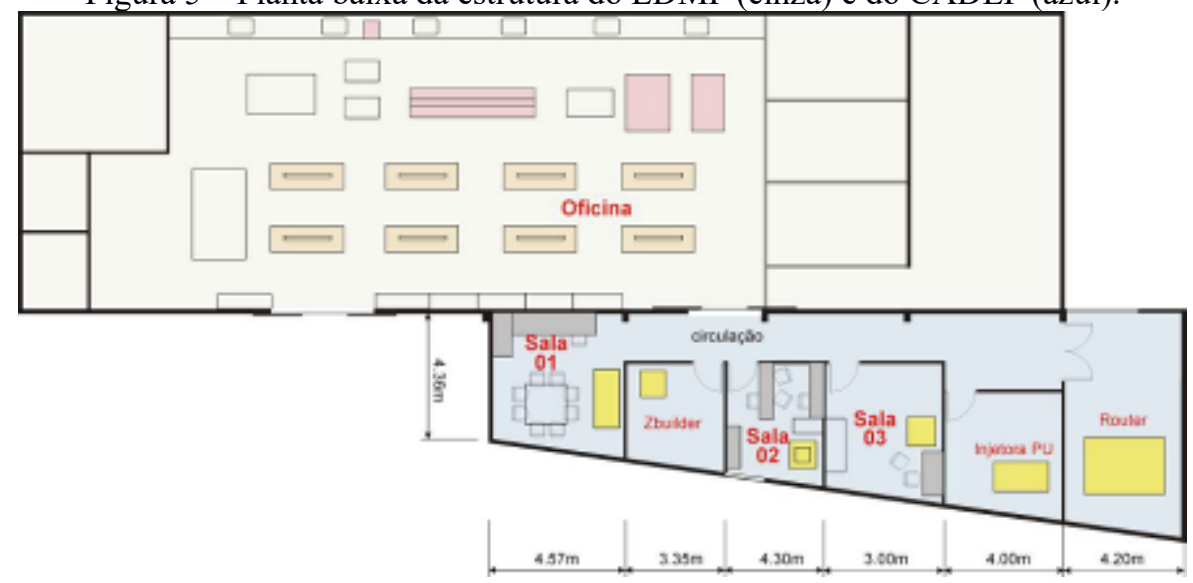

Fonte: Alcoforado, 2014

Na pesquisa, além de permitir um natural incremento das investigações, o grande ganho fica por conta do trabalho conjunto com outras áreas do conhecimento, já que, apesar de terem nascido com foco na indústria em geral, a área da saúde é, atualmente, uma das que mais têm utilizado as tecnologias disponíveis (CADEP, 2017).

\subsubsection{Laboratório de Processamento da Madeira e Bambu}

Utilizado preferencialmente para processamento de Madeira e Bambu, o LPMB (Figura 6) está associado ao Projeto Bambu desde o seu início no ano de 1990 na Unesp - Campus de Bauru. Além da infraestrutura da oficina, o Projeto conta com plantação própria que, desde o ano de 1998, vem produzindo colmos com dimensões adequadas para o processamento e utilização em pesquisas.

Figura 6 - Fotos das instalações do LPMB.

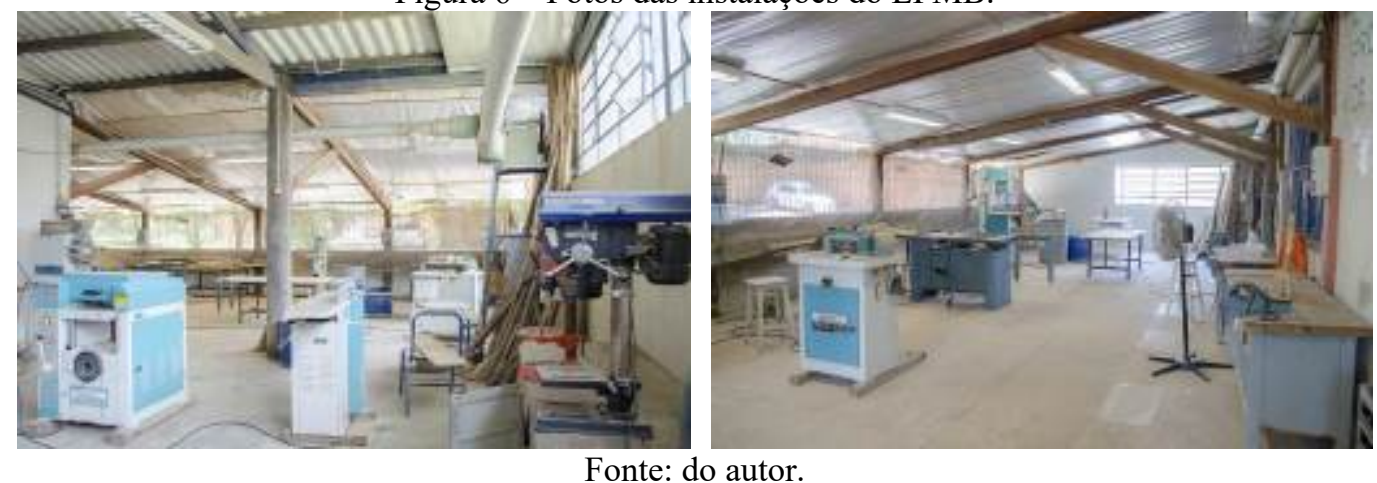

O Laboratório oferece ferramentas convencionais de marcenaria e também específicas para o processamento de espécies prioritárias de bambu (de interesse tecnológico e econômico) com o objetivo de fornecer matéria-prima para as pesquisas que envolvam produção de colmos, 
caracterização física e mecânica dos colmos, processamento, tratamento, desenvolvimento de estruturas leves, de produtos artesanais e processados (bambu laminado colado - BlaC). O projeto atua no ensino (alunos de graduação e pós-graduação) e também em extensão com a comunidade, buscando contribuir para o desenvolvimento da sociedade, da economia e do meio ambiente.

Além da Supertaquara, um equipamento próprio para processamento de lâminas de bambu para a confecção de laminados de bambu, a infraestrutura do laboratório conta com uma Desempenadeira DGR-4 400mm Baldan, uma Desengrossadeira DPC-3 1200 x $250 \mathrm{~mm}$, duas Lixadeiras de Fita LFH-2 530 x 160 mm Baldan, uma Serra de Fita SF-2F Baldan, uma Serra Circular Destopadeira Verry, um Moinho de Facas Marconi, uma Tupia de Mesa TU-3 Baldan, um Triturador de resíduos 2660 TMF, uma Serra Circular Refiladeira dupla, e também ferramentas convencionais como Furadeiras de Bancada, Serra Circular, Moto Esmeril e outros.

\subsubsection{Laboratório de Ergonomia e Interfaces - LEI}

Figura 7 - Logo do Laboratório de Ergonomia de Interfaces da Unesp.

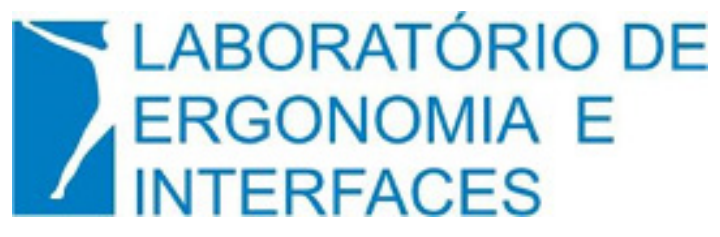

Fonte: FAAC, 2017.

O Laboratório de Ergonomia e Interfaces (LEI) é um ambiente destinado ao estudo e desenvolvimento científico e tecnológico nas áreas de Ergonomia, Usabilidade, Desenvolvimento de Projeto de Produto e Sistemas Informacionais. Trata-se de um laboratório de pesquisa científica, que envolve alunos bolsistas de Iniciação Científica, Mestrandos, Doutorandos e Pós-doutorandos. Atua especialmente nas áreas de ergonomia física e cognitiva, contribuindo no design de produto e informacional. Também desenvolve assessoria para entidades civis, do setor público e privado, com vistas a esclarecer questões, elaborar laudos e contribuir no desenvolvimento de novas tecnologias. É importante destacar que o LEI agrega o Grupo de Pesquisa "Desenho Industrial: Projeto e Interfaces" (FAAC, 2017b).

Além das salas de testes e de estudos, o LEI conta com diversos utensílios para análises ergonômicas. Dentre eles destacam-se os seguintes equipamentos: Torquímetro Estático de 100N, Torquímetro Móvel de 10N, Dinamômetro Digital (AFG), Luva de Distribuição de Pressão na Face Palmar, Eletromiógrafo, Pinch Gauge, Sensores de Movimento, Balança de Precisão bem como computadores com softwares dos equipamentos citados e de análises preditivas como o SPSS.

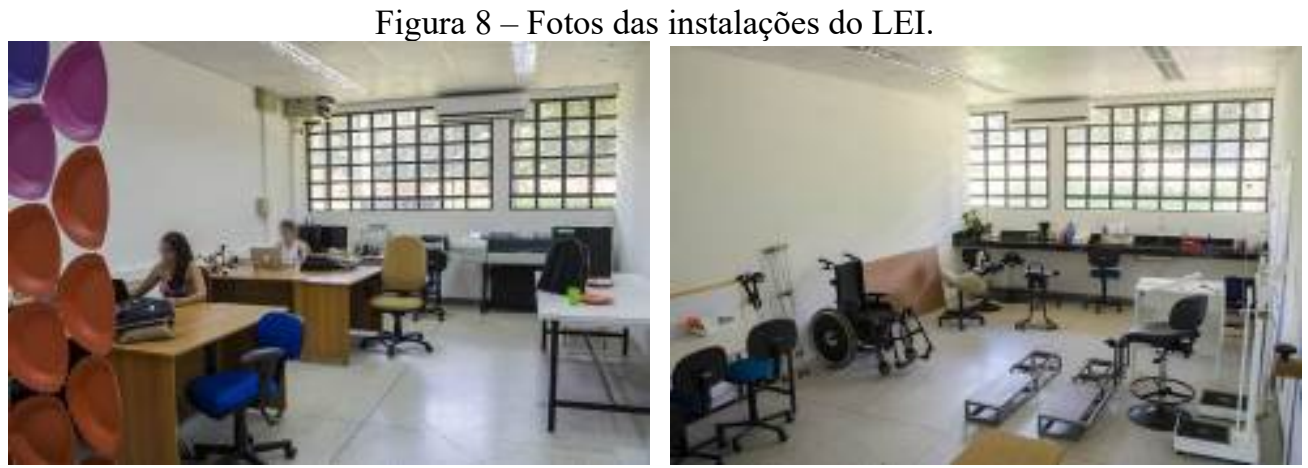

Fonte: do autor. 


\subsubsection{Análise das técnicas e dos materiais}

Desde a criação dos laboratórios abordados neste trabalho, inúmeros modelos e protótipos foram desenvolvidos e testados tanto por alunos de graduação e pós-graduação quanto por professores e técnicos da universidade. Como exemplo, foram selecionados três protótipos cujo processo de produção adotou os dois tipos de prototipagem (convencional e contemporâneo) e são apresentados pela Figura 9. Da esquerda para a direita: 1. Soapbox Alf (RODRIGUES et al., 2014); 2. Descascador Manual (NISHIMURA et al., 2016) e; 3. Prótese Transtibial Endoesquelética Protebam (do autor).

Figura 9 - Fotos da produção dos protótipos selecionados como exemplo.
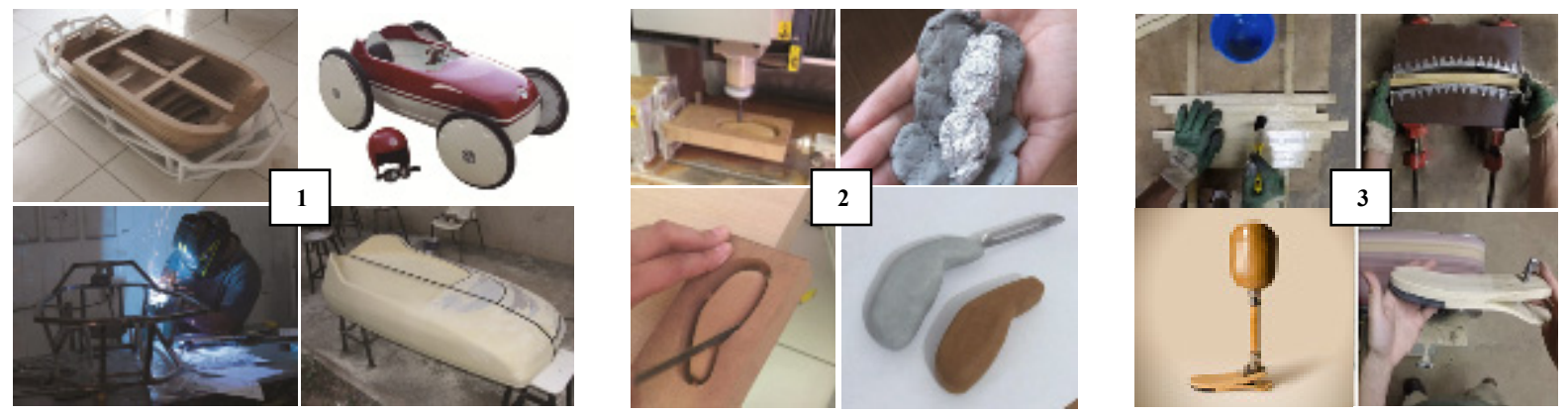

O conjunto 1 da Figura 9 apresenta imagens da produção do protótipo ALF (carro tipo soapbox). Dos materiais utilizados podem-se destacar os tubos de metal de seção quadrada para a estrutura, placas de MDF para confecção do molde e placas de ABS para carroceria. Os processos incluem: soldagem dos tubos, usinagem das placas de MDF e moldagem da carroceria em vacuumforming.

Quanto ao conjunto 2, Nishimura et al. (2016) analisaram os aspectos dos dois tipos de prototipagem através da confecção de dois modelos de Descascador de Alimentos manual para análise ergonômica. As técnicas convencionais foram empregadas na modelagem manual de massa epóxi e papel alumínio (preenchimento), enquanto que, as técnicas contemporâneas foram utilizadas na tecnologia CNC para usinagem de um bloco de MDF conforme modelo virtual paramétrico.

Já o conjunto 3, refere-se à produção de uma prótese transtibial de bambu, intitulada Protebam. Para sua produção foram empregadas técnicas convencionais de processamento e laminação de bambu (BLaC - bambu laminado colado) bem como na produção de um biocompósito com fibras de bambu. As técnicas contemporâneas foram empregadas no projeto através da prototipagem virtual com auxílio de softwares CAD, CAE e CAM (Computer Aided - Design, Engineering e Manufacturing).

Além dos materiais e processos apresentados, diversos outros materiais e processos são utilizados. Os materiais que mais se destacam foram analisados e apresentados na Tabela 1. Para facilitar a leitura, os materiais foram organizados em ordem alfabética com as respectivas técnicas empregadas e avaliadas de acordo com seu Custo, Tempo e Fidelidade do modelo. Para avaliação de Custo e Fidelidade, foram estipuladas cinco escalas de acordo com o nível representado pelo material e técnica utilizada, são eles: Muito Baixo, Baixo, Regular, Alto e Muito Alto. Para avaliar o tempo, as cinco escalas utilizadas foram Muito Lento, Lento, Regular, Rápido e Muito Rápido. O fator Fidelidade representa um conjunto de aspectos referentes à Função Estética (superfície, cor e textura); Função simbólica (fidelidade da forma); e Função Prática (ergonomia e propriedades físicas) resumidas em apenas um termo para facilitar a leitura. Para estabelecimento de um parâmetro, as classificações referentes ao Custo e ao Tempo têm como referência o modelo apresentado pela Figura 10 utilizado no experimento de Pereira (2015).

Figura 10 - Embalagem de cappuccino e Modelo impresso na Z Printer.
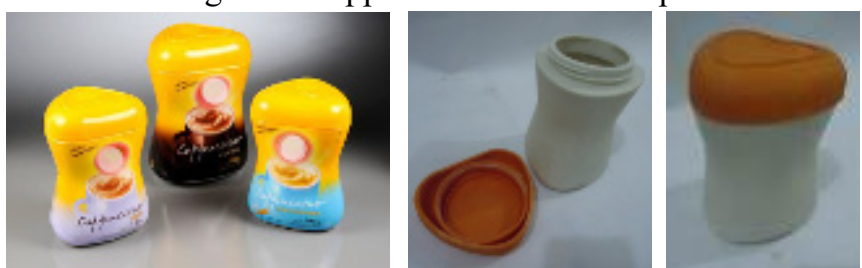

Fonte: Pereira, 2015. 
Tabela 1 - Materiais e técnicas de prototipagem utilizadas nos laboratórios do curso de Design da Unesp de Bauru.

\begin{tabular}{|c|c|c|c|c|c|}
\hline Material & Técnica & Custo & Tempo & Fidelidade & Observações \\
\hline \multirow{3}{*}{ Bambu } & BlaC & Baixo & $\begin{array}{c}\text { Muito } \\
\text { lento }\end{array}$ & Regular & Requer pressão \\
\hline & In Natura & $\begin{array}{l}\text { Muito } \\
\text { Baixo }\end{array}$ & Lento & Regular & $\begin{array}{c}\text { Requer tratamento contra } \\
\text { fatores externos }\end{array}$ \\
\hline & Partículas & $\begin{array}{l}\text { Muito } \\
\text { Baixo }\end{array}$ & Regular & Regular & Reforço para compósitos \\
\hline \multirow{3}{*}{$\begin{array}{c}\text { Cera de } \\
\text { Modelagem }\end{array}$} & Manual & Regular & Rápido & Regular & Relativamente frágil \\
\hline & Usinagem & Alto & Rápido & Muito Alta & Fácil usinagem \\
\hline & Molde & Regular & Regular & Muito Alta & $\begin{array}{c}\text { Para rotomoldagem ou } \\
\text { fundição }\end{array}$ \\
\hline \multirow[t]{2}{*}{ Clay } & Manual & Regular & Regular & Regular & $\begin{array}{c}\text { Requer aquecimento para } \\
\text { moldagem }\end{array}$ \\
\hline & Usinagem & Alto & Baixo & Alta & Pode ser reutilizado \\
\hline \multirow{2}{*}{ Compensado } & Manual & $\begin{array}{l}\text { Muito } \\
\text { Baixo }\end{array}$ & Regular & Baixa & \multirow{2}{*}{$\begin{array}{c}\text { O acabamento geralmente } \\
\text { é grosseiro, necessitando } \\
\text { de outros materiais. }\end{array}$} \\
\hline & Usinagem & Regular & Rápido & Baixa & \\
\hline \multirow{3}{*}{$\begin{array}{l}\text { Espuma de } \\
\text { Poliuretano }\end{array}$} & Manual & Baixo & Lento & Baixa & Frágil e Poroso \\
\hline & Injeção & Regular & Regular & Alta & Requer molde e pressão \\
\hline & Usinagem & Alto & Rápido & Baixa & Acabamento grosseiro \\
\hline Fibra de Carbono & Laminação & $\begin{array}{l}\text { Muito } \\
\text { Alto } \\
\end{array}$ & $\begin{array}{l}\text { Muito } \\
\text { Lento }\end{array}$ & Muito Alta & $\begin{array}{c}\text { Molde positivo ou } \\
\text { negativo e resina }\end{array}$ \\
\hline Fibra de Vidro & Laminação & Alto & $\begin{array}{l}\text { Muito } \\
\text { Lento }\end{array}$ & Alta & $\begin{array}{c}\text { Molde positivo ou } \\
\text { negativo e resina }\end{array}$ \\
\hline \multirow{2}{*}{ Gesso } & Manual & $\begin{array}{l}\text { Muito } \\
\text { Baixo }\end{array}$ & Regular & Baixa & $\begin{array}{c}\text { Acabamento frágil e } \\
\text { poroso }\end{array}$ \\
\hline & Molde & $\begin{array}{l}\text { Muito } \\
\text { Baixo }\end{array}$ & Rápido & Alta & $\begin{array}{c}\text { Molde positivo ou } \\
\text { negativo }\end{array}$ \\
\hline \multirow[t]{2}{*}{ Madeira } & Manual & $\begin{array}{l}\text { Muito } \\
\text { Baixo } \\
\end{array}$ & Lento & Regular & \multirow{2}{*}{$\begin{array}{l}\text { Requer tratamento contra } \\
\text { fatores externos }\end{array}$} \\
\hline & Usinagem & Regular & Rápido & Alta & \\
\hline Massa Plástica & Manual & Baixo & $\begin{array}{l}\text { Muito } \\
\text { Lento }\end{array}$ & Alta & $\begin{array}{c}\text { Geralmente utilizada para } \\
\text { de acabamento }\end{array}$ \\
\hline \multirow{2}{*}{ MDF } & Manual & $\begin{array}{l}\text { Muito } \\
\text { Baixo }\end{array}$ & Rápido & Baixa & \multirow{2}{*}{$\begin{array}{l}\text { Requer cuidados com a } \\
\text { umidade e acabamento }\end{array}$} \\
\hline & Usinagem & Regular & $\begin{array}{l}\text { Muito } \\
\text { Rápido }\end{array}$ & Regular & \\
\hline \multirow{2}{*}{$\begin{array}{c}\text { Metais } \\
\text { (Aço, Alumínio, } \\
\text { etc.) }\end{array}$} & Manual & Alto & $\begin{array}{l}\text { Muito } \\
\text { Lento }\end{array}$ & Regular & \multirow{2}{*}{$\begin{array}{l}\text { Difícil correção e } \\
\text { trabalhabilidade } \\
\text { relativamente complexa }\end{array}$} \\
\hline & Usinagem & $\begin{array}{l}\text { Muito } \\
\text { Alto }\end{array}$ & $\begin{array}{l}\text { Muito } \\
\text { Lento }\end{array}$ & Muito Alta & \\
\hline Papel/Papelão & Manual & $\begin{array}{l}\text { Muito } \\
\text { Baixo }\end{array}$ & $\begin{array}{l}\text { Muito } \\
\text { Rápido }\end{array}$ & Muito Baixa & Ideal para maquetes \\
\hline \multirow[b]{2}{*}{ Poliestireno } & Expandido & Alto & Lento & Muito Baixa & Usado para enchimento \\
\hline & Placa & $\begin{array}{l}\text { Muito } \\
\text { Baixo }\end{array}$ & Rápido & Regular & $\begin{array}{l}\text { Ideal para superfícies } \\
\text { retilíneas ou curvas } \\
\text { suaves (soprador térmico) }\end{array}$ \\
\hline \multirow[b]{2}{*}{ Resina de Poliéster } & Molde & Regular & Lento & Alta & \multirow{2}{*}{$\begin{array}{c}\text { Requer molde positivo ou } \\
\text { negativo }\end{array}$} \\
\hline & $\begin{array}{l}\text { Laminação } \\
\text { (Fibra) }\end{array}$ & Alto & Lento & Regular & \\
\hline Resina Poliuretana & Molde & Médio & Rápido & Alta & Permite rotomoldagem \\
\hline Silicone & Molde & Regular & Regular & Alta & Requer Molde \\
\hline
\end{tabular}


A Figura 11 apresenta as fotos dos equipamentos apresentados na Tabela 2, que, por sua vez, possui uma breve descrição de cada equipamento de prototipagem rápida disponível no CADEP bem como uma análise dos seus aspectos.

Figura 11 - Equipamentos de prototipagem rápida disponíveis no curso de Design da Unesp de Bauru em ordem de aparição da Tabela 2.
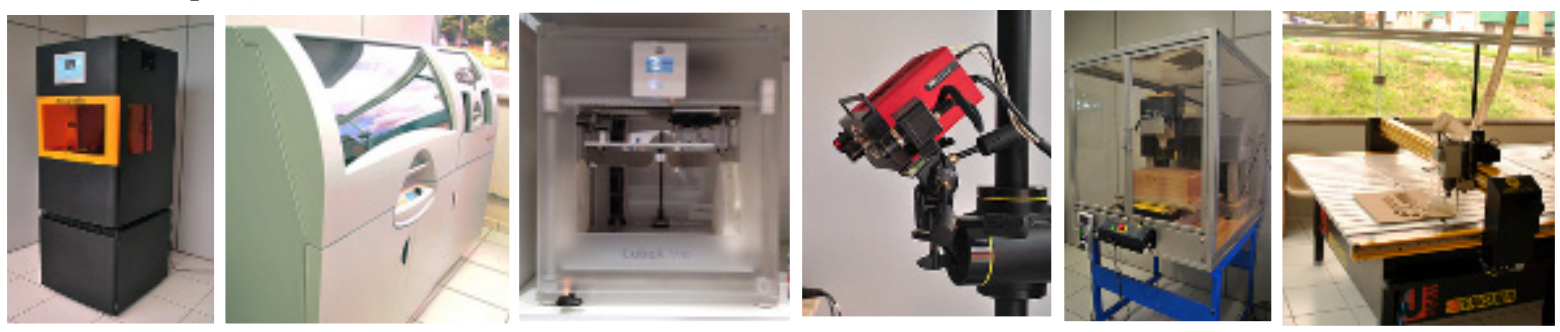

Fonte: CADEP, 2017.

Tabela 2 - Descrição dos equipamentos de prototipagem rápida disponíveis no curso de Design da Unesp de Bauru.

\begin{tabular}{|c|c|c|c|c|c|}
\hline Equipamento & Tecnologia & Custo & Tempo & Fidelidade & Observações \\
\hline $\begin{array}{l}\text { Z-Builder Envision } \\
\text { Ultra }\end{array}$ & $\begin{array}{c}\text { Resina } \\
\text { Fotopolimerizável }\end{array}$ & $\begin{array}{l}\text { Muito } \\
\text { Alto }\end{array}$ & Rápido & Muito Alta & $\begin{array}{l}\text { Excelente } \\
\text { acabamento e } \\
\text { precisão. Permite } \\
\text { realização de } \\
\text { ensaios mecânicos. }\end{array}$ \\
\hline Z-Printer 650 Z-Corp & Deposição de Gesso & Alto & Rápido & Alta & $\begin{array}{l}\text { Protótipos com } \\
\text { partes móveis. } \\
\text { Imprime em cores. }\end{array}$ \\
\hline $\begin{array}{l}\text { 3D Cube X Duo 3D } \\
\text { Systems }\end{array}$ & $\begin{array}{c}\text { Aquecimento e } \\
\text { deposição de ABS } \\
\text { ou PLA }\end{array}$ & Baixo & Rápido & Regular & $\begin{array}{l}\text { Opera com dois } \\
\text { materiais/cores } \\
\text { diferentes } \\
\text { simultaneamente } \\
\end{array}$ \\
\hline $\begin{array}{c}\text { Scanner } \\
\text { tridimensional ótico } \\
\text { 3D GOM Atos I 2M }\end{array}$ & Escaneamento 3D & \multicolumn{4}{|c|}{$\begin{array}{c}\text { O escaneamento gera uma "nuvem" de pontos ou uma malha } \\
\text { poligonal virtual que pode ser aplicada na Prototipagem Rápida, } \\
\text { no Controle de Qualidade, na Engenharia Reversa ou na } \\
\text { Inspeção Tridimensional, sem equipamentos adicionais. Mede } \\
\text { objetos de até } 20 \mathrm{~m} \text {. }\end{array}$} \\
\hline $\begin{array}{l}\text { CNC Roland MDX } \\
540 \text { com } 4^{\circ} \text { eixo } \\
\text { rotacional }^{5}\end{array}$ & $\begin{array}{c}\text { Subtração de } \\
\text { Material } \\
\text { (de plásticos a } \\
\text { metais não ferrosos) }\end{array}$ & Regular & Rápido & Muito Alta & $\begin{array}{l}\text { Não necessita } \\
\text { acabamento }\end{array}$ \\
\hline $\begin{array}{c}\text { CNC 3D Transform } \\
2000 / 2\end{array}$ & $\begin{array}{c}\text { Subtração de } \\
\text { Material } \\
\text { (de plásticos a } \\
\text { metais). } \\
\end{array}$ & Regular & Rápido & Regular & $\begin{array}{l}\text { Permite trabalhar } \\
\text { com chapas } \\
\text { inteiras de MDF }\end{array}$ \\
\hline
\end{tabular}

Fonte: do autor.

\section{Considerações finais}

Através das análises realizadas, concluiu-se que as técnicas convencionais de prototipagem que incluem a modelagem manual e materiais convencionais (madeira, massa plástica, etc.) são mais apropriadas para modelos de baixa fidelidade com baixo custo e que precisam ser experimentados durante o processo de modelagem. Enquanto que a prototipagem rápida, por proporcionar maior precisão e melhor acabamento, indicada para produtos sofisticados que necessitem de exatidão, tanto nas medidas quanto na fabricação, que não se alcança através de ferramentas manuais. Logo, deduz-se que, quando viável em termos de custo e tempo, a adoção das duas técnicas é a maneira mais eficaz 
para se desenvolver modelos e protótipos. Sendo as técnicas de baixo custo preferencialmente adotadas no início do projeto, por ser a etapa onde são realizadas mudanças frequentes e drásticas. Enquanto que as técnicas sofisticadas são mais indicadas para as fases finais da prototipagem, quando já não há mudanças radicais na configuração do produto e quando há necessidade de um melhor acabamento e fidelidade com o produto final.

Com a reunião de dados referentes aos laboratórios, suas infraestruturas, equipamentos disponíveis e materiais comumente empregados, foi possível organizar um material informativo de caráter didático que facilita o acesso à informação referente à infraestrutura do curso e auxilia alunos, pesquisadores e interessados nas decisões referentes aos seus projetos.

Dado o caráter subjetivo das avaliações realizadas e a grande quantidade de fatores variáveis como a qualidade dos materiais, a experiência do projetista, o tipo de ferramentas e outros aspectos que influenciam na qualidade final do protótipo, a avaliação dos materiais e técnicas apresenta apenas uma análise superficial das características dos materiais e dos processos apresentados. É importante ressaltar que tais características podem divergir de acordo com o produto a ser confeccionado. Portanto, cabe ao responsável do projeto a análise precisa dos fatores relacionados à etapa de prototipagem do produto em questão e seleção das técnicas compatíveis e indicadas com as especificações de seu projeto.

\section{Description and Analysis of Prototyping Techniques in the Design course of Unesp of Bauru}

Abstract: Virtual prototyping has revolutionized the Product Development Process, enabling the production of complex and well-detailed physical parts through virtual models, achieving great advantages mainly in reducing time and innovation. In the academic world these virtual modeling softwares come slowly due to the lack of infrastructure, resulting in students with lagged knowledge. New prototyping techniques, as well as conventional techniques, are important for the development of professionals in the areas related to innovation and development of new products. Therefore, the purpose of the present work is to develop a material that serves as a reference for professionals and students of the area. For that, a research was carried out regarding the conventional and contemporary methods and materials of modeling and prototyping of the Design course of Faculdade de Arquitetura Artes e Comunicação - UNESP, Campus of Bauru. Through a consultation of available bibliographical materials, teachers and technicians working in the sector, it was carried out a documentary collection of equipment, processes and data about the methods and materials used for modeling and prototyping, obtaining an overview of the current infrastructure of laboratories, techniques and technologies used in the course and their particularities. Since it is a research focused on the design of products, only the laboratories related to the development of models and prototypes were approached, being: Didactic Laboratory of Materials and Prototypes (LDMP), the Advanced Center for Product Development (CADEP), the Laboratory of Wood and Bamboo Processing (LPMB) and the Laboratory of Ergonomics and Interfaces (LEI). The results of this documentary collection have been organized into topics that follow a chronological order from the foundation of the University's Design course, the creation of the laboratories and finishing with the description of the methodologies and prototyping techniques used.

Keywords: Rapid prototyping; 3D printing; Design; Unesp; Bauru 


\section{Referências bibliográficas}

ALCOFORADO, M. G. Metodologia de Design Mediada por protótipos [Tese de Doutorado] Bauru: Unesp, 2014, 460 p.

BARATA, T. Q. F. ; RODRIGUES, Osmar Vicente . A Atividade projetiva no Ensino de Design: Estudo de caso. Ensaios em Design: Pesquisa e Projetos. 1ed.Bauru: canal 6, 2013, v. 1, p. 42-61.

CADEP, Centro Avançado de Desenvolvimento de Produtos. Disponível em: $<$ www.faac.unesp.br/\#!/departamentos/design/laboratorios-de-pesquisa-e-extensao/cadep/>. Acesso em 10/01/2017.

FAAC, Histórico da Faculdade de Arquitetura, Artes e Comunicação. Disponível em: <www.faac.unesp.br/\#!/instituicao/historico/> Acesso em 10/03/2017a.

FAAC, Laboratórios do Curso de Design da Unesp - Campus de Bauru Disponível em: <http://www.faac.unesp.br/\#!/departamentos/design/laboratorios/> Acesso em 10/03/2017b.

FORTI, F. S. A. Uma Avaliação do ensino da Prototipagem Virtual nas Graduações de Design de Produto do Estado do Rio de Janeiro. [Dissertação de Mestrado], COPPE, Universidade Federal do rio de Janeiro, 2005, 105p.

GRIMM, T. Virtual Versus Physical: will computer-generated virtual prototypes obsolete rapid prototyping? Time-Compression Technologies, v. 13, 2005.

KNIGHT, T. Interaction in Visual Design Computing. Massachusetts Institute of Technology, Cambridge, Massachusetts, EUA, 2004 p.1-31

LAKATOS, E. M. e MARCONI, M. A. Metodologia Científica. 2ª Edição. S. Paulo: Ed. Atlas, 1995.

LDMP, Laboratório Didático de Materiais e Protótipos. Disponível em: <www.faac.unesp.br/\#!/departamentos/design/laboratorios/ldmp/>. Acesso em 10/01/2017.

LOPES, J.; NAVEIRO, R. M.; O Uso de Modelos Tridimensionais no Desenvolvimento de Projetos. Anais do $3^{\circ}$ Congresso Brasileiro de Pesquisa e Desenvolvimento em Design, Rio de Janeiro, 1998.

NISHIMURA, P. L. G.; KICK, P. G. S.; RODRIGUES, O. V.; BOTURA JUNIOR, G. ; PASCHOARELLI, L. C. A Prototipagem Rápida aplicada à avaliação ergonômica: estudo comparativo entre um mock-up produzido manualmente e outro obtido por uma fresadora CNC. In: V CONGRESSO ERGOTRIP DESIGN 2016, 2016, Natal/RN. Proceedings of V Congresso Ergotrip Design, 2016.

PEREIRA, D. D. O uso da modelagem aplicada à ergonomia no desenvolvimento de produtos. 176 f. Dissertação (mestrado) - Universidade Estadual Paulista Julio de Mesquita Filho, Faculdade de Arquitetura, Artes e Comunicação, 2015.

RODRIGUES, O. V.. Modelagem de Veículos Utilizando Espuma Rígida de Poliuretano Expandido. Ensaios em Design: Ensino e Produção de Conhecimento. 1ed.Bauru SP: Canal 6, 2011, v. 1 , p. $128-157$.

RODRIGUES, O. V.; ALENCAR, F.; BARATA T. Q. F. Combining Rapid Prototyping with more conventional production processes. In: 5th International PMI Conference Proceedings. Guent: University College Guent, 2012. V. 1, p. $147-150$.

RODRIGUES, O. V.; OLIVEIRA, C. I. M.; ANTONIUCCI G. M. Concurso Talento Design Vw 2013 Projeto Soapbox ALF. Ensaios em Design: Práticas interdisciplinares. 1ed.Bauru: canal 6, 2014, v. 1, p. 178-202. 2014.

ROZENFELD, H. et al. Gestão de Desenvolvimento de Produtos - Uma referência para a melhoria do processo. São Paulo, Saraiva, 2006.

SÃO PAULO (SP) Decreto N. 28.682, de 15 de Agosto de 1988 . Diário Oficial Estado de São Paulo, v. 98, no153, p. 1, ago. 1988. Disponível em: <http://www.al.sp.gov.br/norma/?id=34010>. Acesso em 10/01/2017. 